\title{
ARTICLE
}

Cellular and Molecular Biology

\section{HOX genes promote cell proliferation and are potential therapeutic targets in adrenocortical tumours}

\author{
Jeffrey C. Francis ${ }^{1}{ }^{1}$, Jennifer R. Gardiner ${ }^{1}$, Yoan Renaud ${ }^{2}$, Ritika Chauhan ${ }^{3}$, Yacob Weinstein ${ }^{4}$, Celso Gomez-Sanchez ${ }^{5}$, \\ Anne-Marie Lefrançois-Martinez ${ }^{2}$, Jérôme Bertherat ${ }^{6}$, Pierre Val $^{2}$ and Amanda Swain (iD)
}

BACKGROUND: Understanding the pathways that drive adrenocortical carcinoma (ACC) is essential to the development of more effective therapies. This study investigates the role of the transcription factor HOXB9 and other HOX factors in ACC and its treatment.

METHODS: We used transgenic mouse models to determine the role of Hoxb9 in adrenal tumour development. Patient transcriptomic data was analysed for the expression of HOX genes and their association with disease. Drug response studies on various adrenocortical models were done to establish novel therapeutic options.

RESULTS: Our human ACC dataset analyses showed high expression of HOXB9, and other HOX factors, are associated with poorer prognosis. Transgenic overexpression of Hoxb9 in the adrenal cortex of mice with activated Ctnnb1 led to larger adrenal tumours. This phenotype was preferentially observed in male mice and was characterised by more proliferating cells and an increase in the expression of cell cycle genes, including Ccne1. Adrenal tumour cells were found to be dependent on HOX function for survival and were sensitive to a specific peptide inhibitor.

CONCLUSIONS: These studies show Hoxb9 can promote adrenal tumour progression in a sex-dependent manner and have identified HOX factors as potential drug targets, leading to novel therapeutic approaches in ACC.

British Journal of Cancer (2021) 124:805-816; https://doi.org/10.1038/s41416-020-01166-z

\section{BACKGROUND}

Tumours of the adrenal cortex are relatively common with a prevalence of $1-10 \%$. Most of these are benign adenomas, however, in rare cases (up to 2 per million per year) adrenocortical carcinoma (ACC) can develop, which is an aggressive disease with a low 5 -year survival (up to $35 \%$ of diagnosed patients and less than $15 \%$ in patients with metastatic disease) (reviewed in refs. ${ }^{1,2}$ ). Current treatments include surgery for non-metastatic disease and mitotane therapy, which has shown limited efficacy in advanced patients. Understanding the pathways that drive ACC progression is essential to the development of more effective treatments and for the prediction of individual outcomes.

Relatively little is known about the molecular pathways that drive ACC growth and progression. Recent next-generation sequencing studies have revealed recurrent alterations present in patients with $A C C .^{3-5}$ These studies have identified mutations in the WNT signalling pathway to be one of the most frequent alterations, with CTNNB1-activating mutations present in up to $16 \%$ and inactivating changes in ZNRF3, a pathway repressor, in up to $21 \%{ }^{3}$ Other pathways that are altered in ACC patients include epigenetic regulation and p53/RB1 and PKA signalling. ${ }^{5}$
Studies in mice have shown that targeting an activating mutation in Ctnnb1 to the adrenal cortex leads to tumour formation, highlighting the relevance of this model to study this disease. ${ }^{6}$ WNT signalling has been implicated in adrenal development and homoeostasis. Genetic studies in mice have shown that a decrease in WNT signalling, either through deletion of Ctnnb1 or pathway inhibition, in adrenal cortical cells led to adrenal aplasia, degeneration and zonation defects, depending on the deletion stage and cell type. ${ }^{7-9}$ These studies also indicate the role of WNT signalling in subcapsular stem/progenitor cell proliferation and maintenance. $^{10}$

Factors involved in organ development have been implicated in neoplasia in many tissues. HOX genes are a family of homeobox transcription factors that are major regulators of embryogenesis and have been implicated in oncogenesis. ${ }^{11}$ In mammals there are four clusters of HOX genes (HOXA, HOXB, HOXC and HOXD) with 39 members identified in humans. Gene expression studies have shown that several HOX genes are expressed in the developing and adult adrenal glands, while the HOX cofactor PBX1 is required for adrenal formation. ${ }^{12-15}$ Emerging evidence has revealed that aberrant HOX gene expression in adult tissues can lead to malignancy, including in lung, ovarian, cervical, prostate and

\footnotetext{
${ }^{1}$ Division of Cancer Biology, The Institute of Cancer Research, 237 Fulham Road, London, UK; ${ }^{2}$ Genétique Reproduction \& Développement, CNRS UMR 6293, Inserm U1103, Université Clermont Auvergne, 63001 Clermont-Ferrand, France; ${ }^{3}$ Tumour Profiling Unit, The Institute of Cancer Research, 237 Fulham Road, London, UK; ${ }^{4}$ The Shraga Segal Dept. of Microbiology, Immunology and Genetics, Faculty of Health Sciences, Ben Gurion University of the Negev, Beer Sheva 84105, Israel; ${ }^{5}$ Division of Endocrinology, Medical Service,

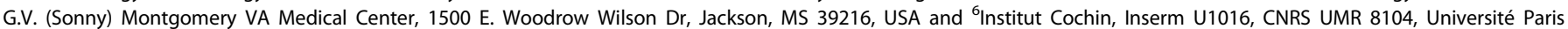
Descartes, UMR-S1016, 75014 Paris, France

Correspondence: Amanda Swain (amanda.swain@icr.ac.uk)
}

Received: 4 February 2020 Revised: 23 September 2020 Accepted: 29 October 2020

Published online: 20 November 2020 
breast carcinoma, ${ }^{16-21}$ and can interact with other signalling pathways, such as WNT/ $\beta$-catenin, to promote tumour progression. ${ }^{22-26}$

Here, we show that high expression of HOXB9, which is expressed in adrenal cortical cells during development, is associated with patient survival in ACC. A genetic mouse model of overexpression of Hoxb9 in adrenal cortical cells shows a bigger $\mathrm{X}$ zone. Male mice with an activating Ctnnb1 mutation and Hoxb9 overexpression develop tumours with increased proliferation. Our study implicates HOX genes in ACC formation and identifies them as possible drug targets in this disease.

\section{METHODS}

Mouse strains

The mutant Ctnnb1 mice were generated by breeding two previously described strains, Cyp11a1:Cre and the stabilised Ctnnb1 conditional allele. ${ }^{27,28}$ The Sf-1:Hoxb9 transgenic mice were generated by injecting a BAC construct into one-cell mouse embryos, as described previously. ${ }^{27}$ The BAC construct was generated by inserting, through homologous recombination, the mouse Hoxb9 cDNA plus the bovine growth hormone polyadenylation signal sequences into the Sacll site in the $5^{\prime}$ untranslated region of the mouse $S f-1$ locus contained in a BAC plasmid, as described previously. ${ }^{27}$

All animals were bred on a mixed genetic background. All mouse work was carried out in accordance with the Institute of Cancer Research guidelines and with the UK Animals (Scientific Procedures) Act 1986. Animals were housed in a specific pathogen-free environment using an Optimouse system (air speed $4.3 \mathrm{~m} / \mathrm{s}$ average, a 12/12 light cycle (7:30-19:30), temperature $21+/-1{ }^{\circ} \mathrm{C}$, room humidity $\left.55 \%+/-10 \%\right)$, and fed lab diet 5002 (International Product Supplies), with corn-cob bedding 1014 (International Product Supplies), and with card tunnel and woodblock enrichment. Animals were euthanised by exposure to carbon dioxide or isoflurane followed by cervical dislocation. At least four animals of each sex were analysed for each genotype, using animals from the same litters as controls.

Immunohistochemistry (IHC)

Antibody staining was performed as previously described (details in the Supplementary Methods section). For adrenal sections, staining was done on samples from at least three mice of the same genotype.

Western blot

Cells were lysed in RIPA buffer (Sigma, Welwyn Garden Cit, UK) with protease and phosphatase inhibitors (Cell Signaling Technology, Leiden, The Netherlands). Samples were run on $4-12 \%$ BisTris protein gels with MOPS buffer and transferred to nitrocellulose membranes. The membrane was blocked in $5 \%$ milk/TBST (TBS, $0.1 \%$ Tween-20) for $1 \mathrm{~h}$, and primary antibodies incubated in $2.5 \%$ milk/TBST overnight at $4{ }^{\circ} \mathrm{C}$. Membranes were washed with TBST, incubated with HRP-conjugated secondary antibodies for $1 \mathrm{~h}$ at room temperature, washed again and chemiluminescence detected (GE Healthcare, Chalfont St Giles, UK). Primary antibodies used were; HOXB9 (Santa Cruz sc-398500), Sf-1 (Abcam ab65815) and Vinculin (Sigma V4505).

\section{Quantification of cell proliferation and cell death}

The number of proliferating cells was calculated by counting the number of nuclear Ki67-stained cells and shown as a percentage of the total number of cells stained with nuclear haematoxylin. Apoptotic cell death of mouse tissue was quantified by IHC stains of sections with an antibody against active Caspase 3. Cells were counted from at least four high-power randomly selected fields per animal. Three animals of each genotype were analysed. An ANOVA or $t$ test was used to test if there was a significant difference in the number of proliferating or apoptotic cells between each group.

In vitro culture of adrenal cells H295R cells, a kind gift from Peter King (Queen Mary, University of London), were STR profiled to confirm their identity and were maintained in DMEM/F12 supplemented with $2.5 \%$ Nu-Serum I, ITS +1 and GlutaMAX. ATC 1 and ATC7 cells were established from adrenal tumours from transgenic mice with Large $T$ antigen of SV40 controlled by the cortical adrenal-specific Akr1b7 promoter, and were a kind gift from Antoine Martinez. ${ }^{29}$ ATC1 and ATC7 cells were maintained in DMEM/F12 supplemented with 2.5\% FBS, $2.5 \%$ horse serum and ITS. PC3 cells were maintained in RPMI1640 with $10 \%$ FBS. All cell lines were used at low passage number $(<20)$. $A B C$ cells were derived from a mouse Ctnnb1 mutant adrenal gland tumour and grown in Y-media (DMEM/F12, 10\% FBS, penicillin/streptomycin, L-Glutamine, $5 \mu \mathrm{g} / \mathrm{ml}$ Insulin, $0.4 \mu \mathrm{g} / \mathrm{ml}$ hydrocortisone, $10 \mathrm{ng} / \mathrm{ml} \mathrm{EGF,} 10 \mu \mathrm{g} / \mathrm{ml}$ Gentamicin, $250 \mathrm{ng} / \mathrm{ml}$ amphotericin, $4.81 \mathrm{ng} / \mathrm{ml}$ Cholera toxin, $5 \mu \mathrm{M}$ Y27632). Briefly, adrenal glands were dissected, minced and incubated in dissociation media (DMEM/F12, ITS, $10 \mu \mathrm{g} / \mathrm{ml} \mathrm{EGF,} 10 \mathrm{mg} / \mathrm{ml}$ hydrocortisone, $0.5 \mathrm{mg} / \mathrm{ml}$ collagenase, $0.1 \mathrm{mg} / \mathrm{ml}$ hyaluronidase, 100 units/ml DNase I, $10 \mu \mathrm{M}$ Y27632) for $2 \mathrm{~h}$. The cells were washed, incubated in $0.05 \%$ Trypsin/EDTA with $10 \mu \mathrm{M}$ Y 27632 for $8 \mathrm{~min}$ at $37^{\circ} \mathrm{C}$ and dissociated by vigorous pipetting and trypsin inactivated with Y-media. Cells were incubated in $1 \mathrm{mg} / \mathrm{ml}$ DNase for $5 \mathrm{~min}$ at $37^{\circ} \mathrm{C}$, washed with PBS and filtered through $70-\mu \mathrm{m}$ filter.

siRNA knockdown in H295R cells

To perform gene knockdown, $6 \times 10^{5} \mathrm{H} 295 \mathrm{R}$ cells were seeded in six-well plates and the next day siRNA reagents non-targeting ONTARGETplus SMARTpool (D-001810-10-05, Horizon Discovery, Cambridge, UK), PBX1 ON-TARGETplus SMARTpool (L-019680-000005), HOXA10 ON-TARGETplus SMARTpool (L-006336-00-0005), HOXA11 ON-TARGETplus SMARTpool (L-012108-00-0005), or HOXA13 ON-TARGETplus SMARTpool (L-011052-00-0005) were transfected with RNAiMAX (Thermo Fisher Scientific, Hemel Hempstead, UK) following the manufacturer's protocol. Cells were incubated for a further $24 \mathrm{~h}$ and re-plated for the cell growth assay or $48 \mathrm{~h}$ for RNA extraction. For the growth assay, 2000 cells were plated per well of a 96-well plate and CellTitre-Glo luminescence (Promega, Southampton, UK) measurements were taken at the indicated days.

Lentiviral overexpression of HOXB9

H295R cells overexpressing HOXB9 were made by transduction with lentiviral particles generated from pLenti-GIII-CMV-HOXB9GFP-2APuro (LV183492, Applied Biological Materials, Richmond, Canada) and control cells with pLenti-CMV-GFP-2A-Puro (LV590, Applied Biological Materials). Lentivirus was produced by transfecting $7 \times 10^{6} 293$ T cells in $10-\mathrm{cm}$ plates with the packaging plasmids psPAX2 and pMD2.G using Lipofectamine 3000 (Thermo Fisher Scientific). Viral supernatants were collected 24- and 48-h post-transfection, centrifuged at $2000 \mathrm{rpm}$, and filtered through a 45- $\mu \mathrm{m}$ filter.

Generation of a HOX-targeting peptide

Genscript Biotech (Leiden, The Netherlands) synthesised a peptide targeting the HOX-PBX protein interaction and a control peptide, using sequences based on previous publications. ${ }^{30}$ The targeting HTL001 peptide sequence WYKWMKKAARRRRRRRRR and the CXR9 control peptide WYPAMKKHHRRRRRRRRR, both with D-isomer modification of $\mathrm{C}$ - and $\mathrm{N}$-terminals were dissolved in DMSO.

Cell viability assay

Peptide drug survival assays were performed in 96-well plates. PC3 cells were plated at 500 cells per well; H295R, ATC1, ATC7 and ABC 
cells were plated at 2000 cells per well. Drug was added $24 \mathrm{~h}$ after seeding, and cells were continuously exposed to the drug for 5 days, after which cell viability was estimated using CellTitre-Glo luminescence (Promega).

\section{Apoptosis assay}

H295R cells were plated in a 96-well plate at 10,000 cells per well. The next day the IC $\mathrm{C}_{50}$ concentration of $5 \mu \mathrm{M}$ HTL001, $5 \mu \mathrm{M}$ CXR9 or DMSO was added, incubated for $24 \mathrm{~h}$ and apoptosis estimated using the Caspase 3/7 Glo assay (Promega) following the manufacturer's protocol.

\section{RNA isolation and quantitative RT-PCR}

RNA was isolated using the RNeasy kit (Qiagen, Manchester, UK) and cDNA was made using 500 ng RNA with SuperScript IV reverse transcriptase (Thermo Fisher Scientific), following the manufacturer's instructions. Quantitative RT-PCR was carried out using Taqman gene expression assays (probe sequence in the Supplementary Methods section). Experiments on animal tissue were done from at least 3 mice of the same genotype.

\section{Transcriptomic analysis}

For RNA-seq analysis of adrenal tumours, mRNA libraries for were prepared using $500 \mathrm{ng}$ of total RNA of each sample with NEBNext Ultra II Directional RNA Library Prep kit (E7760, NEB, Hitchin, UK) and NEBNext Poly(A) mRNA magnetic beads (E7490, NEB) following the manufacturer's protocol. RNA samples were run on two lanes of a HiSeq2500 using RAPID onboard clustering and single read 50 cycles. All samples were run at $16.6 \%$ per lane to achieve 25 million clusters. TopHat RNA-seq alignment software (v2.1.0) was used to align reads to the reference mouse genome (GRCm38). Once the reads were aligned, HTSeq-count (HTSeq v0.6.1) was used to count the number of reads mapping unambiguously to genomic features in each sample. The raw count data were normalised to correct for library size and RNA composition bias. Differential gene expression analysis of the count data was performed in $\mathrm{R}$ using the Bioconductor package DESeq2 (v1.18.1). Gene set enrichment analysis (GSEA) was carried out with male Ctnnb1 mutant and double-mutant RNA-seq data using the Broad Institute Java plug-in with the Hallmarks gene set. $^{31}$ STRING protein network analysis was carried out using the differentially expressed genes between male Ctnnb1 mutants and double mutants (genes with adjusted $P<0.1$ and $P<0.05$ ).

\section{Patients cohort datasets}

The clinical datasets used in this paper are derived from two cohorts of patients. Cochin's cohort (49 ACC, 101 ACA and 4 NAd, GSE49280) was analysed by Affymetrix microarray (HG-U133 Plus 2.0). ${ }^{3}$ Our collaborators provided data analysed after RMA normalisation and inter-array normalisation. The other transcriptome (79 patients) is from the TCGA database (The Cancer Genome Atlas) and it relies on Illumina HiSeq 2000 RNA Sequencing Version 2. ${ }^{5}$ Expression data were standardised by the Relative Standard Error of the Mean (RSEM) algorithm and transformed into Log2 in order to refocus and symmetrise values' distribution. For each cohort, patients' survival data were available and distribution in the good $(\mathrm{C} 1 \mathrm{~B})$ and poor prognosis $(\mathrm{C} 1 \mathrm{~A})$ groups, which was defined by our collaborators on the basis of unsupervised clustering. Gene proliferation signature was extracted from Wassef et al. ${ }^{32}$ (Supplementary Table S1) and was defined as the mean expression of the 61 genes that compose it. Statistical analyses were performed in R using the ImerTest_3.10 and Ismeans_2.30-0 libraries. Paired comparisons (C1A vs C1B) were analysed by a Wilcoxon test. Multiple comparisons (NAd, ACA and ACC) were analysed by ANOVA followed by Tukey's test. Overall and disease-free survival analyses were conducted with $\mathrm{R}$ package " survival_2.44-1.1 » and displayed as Kaplan-Meier Curves. Pearson correlations were computed using R package «
Hmisc_4.2-0 $\gg$. Graphs were generated using R library « ggplot2_3.1.1 》.

\section{RESULTS}

Hoxb9 overexpression in adrenal cortical cells

HOX genes have been implicated in the initiation and progression of many cancers. ${ }^{11}$ Our and other studies have shown that Hoxb9 is expressed at the early stages of adrenal cortical development. ${ }^{14,33}$ To investigate if HOXB9 expression is associated with ACC, we analysed patient gene expression data from the Cochin cohort that contains normal adrenal (NAd), adrenocortical adenoma (ACA) and ACC samples. ${ }^{3}$ In this dataset, HOXB9 expression was higher in ACC samples with the difference with ACA being significant, but not with NAd (Fig. 1a). Consensus clustering of mRNA expression has been used to subgroup ACC patients into those that have aggressive disease, $\mathrm{C} 1 \mathrm{~A}$, and those with the indolent disease, C1B. In both the Cochin dataset and in ACC samples from The Cancer Gene Atlas (TCGA), HOXB9 expression was significantly higher in C1A compared to C1B (Fig. 1b). Consistent with HOXB9 expression being associated with aggressive disease, analyses of TCGA and Cochin patients into high and low HOXB9 expression showed that ACC patients with high HOXB9 expression had a poorer survival prognosis (Fig. 1c). Together these data suggest that elevated HOXB9 expression in ACC may play a role in tumour progression to aggressive disease.

To investigate the effect of high levels of HOXB9 in adrenal cortical cells, we generated transgenic mice carrying a BAC construct that contained the Hoxb9 cDNA inserted into the Sf-1 locus (Sf-1:Hoxb9 mice, referred to as Hoxb9 t/g, Fig. 1d). Quantitative RT-PCR (qRT-PCR) expression analysis showed an increase of Hoxb9 levels in the adult adrenals of transgenic mice (Fig. 1e). This was confirmed in antibody staining studies, which showed an expanded domain of HOXB9 expression, reflecting the pattern of the Sf-1 promoter sequence driving Hoxb9 (Fig. If and Supplementary Fig. S1A). In the normal adrenal HOXB9 was primarily expressed in zona fasciculata (ZF), while in transgenic animals expression was also found in the outer cortical zona glomerulosa (ZG) cells. HOXB9-expressing cells were also found in the medulla of transgenic glands from 3-month-old female mice that were not present in control animals (Supplementary Fig. S1B). Phenotypic analysis of the adrenals of transgenic mice at 3 and 18 months of age showed no obvious changes in size or structure compared to wild-type adrenal glands (Fig. 1g, h, Supplementary Fig. S1C, D). Ki67 staining revealed no difference in the number of proliferating cells between transgenic and wild-type animals (Fig. 1i, j and Supplementary Fig. S1E). We next analysed the expression of a known embryonic HOX target gene, $S f-1$, markers of adrenal gland stem/progenitor cell function, and a WNT signalling target. In both female and male adrenal glands from transgenic mice, Sf-1 expression was increased threefold, while there was no change in the expression of Shh, Patched (Ptch) or Axin2 (Fig. 1k and Supplementary Fig. S1F). This suggests that elevated Hoxb9 is not able to induce neoplastic development but can promote Sf- 1 expression in the adult gland. To determine if adrenal gland zonation was disrupted by elevated Hoxb9 expression, we performed immunohistochemical (IHC) staining and qRT-PCR analyses with cell-type markers for ZG (Dab2 and Cyp11b2), ZF (Cyp11b1), adrenal medulla (TH) and X zone (20aHSD, gene name $A k r 1 c 18$, and $P i k 3 c 2 g$ ) on male and female adrenals (Fig. 2). These data showed that transgenic animals had no change in adrenal cortical ZG or ZF markers (Fig. 2a, b). Instead, glands from 3-month-old female transgenic mice had a bigger foetal derived $X$ zone with Sf-1-positive cells infiltrating the medulla (Fig. 2c, d and S2A). The larger $X$ zone in transgenic animals behaved as in the wild-type in that it was only found in prepubertal males (Supplementary Fig. S2B) and it regressed in older females (Fig. 2c). 

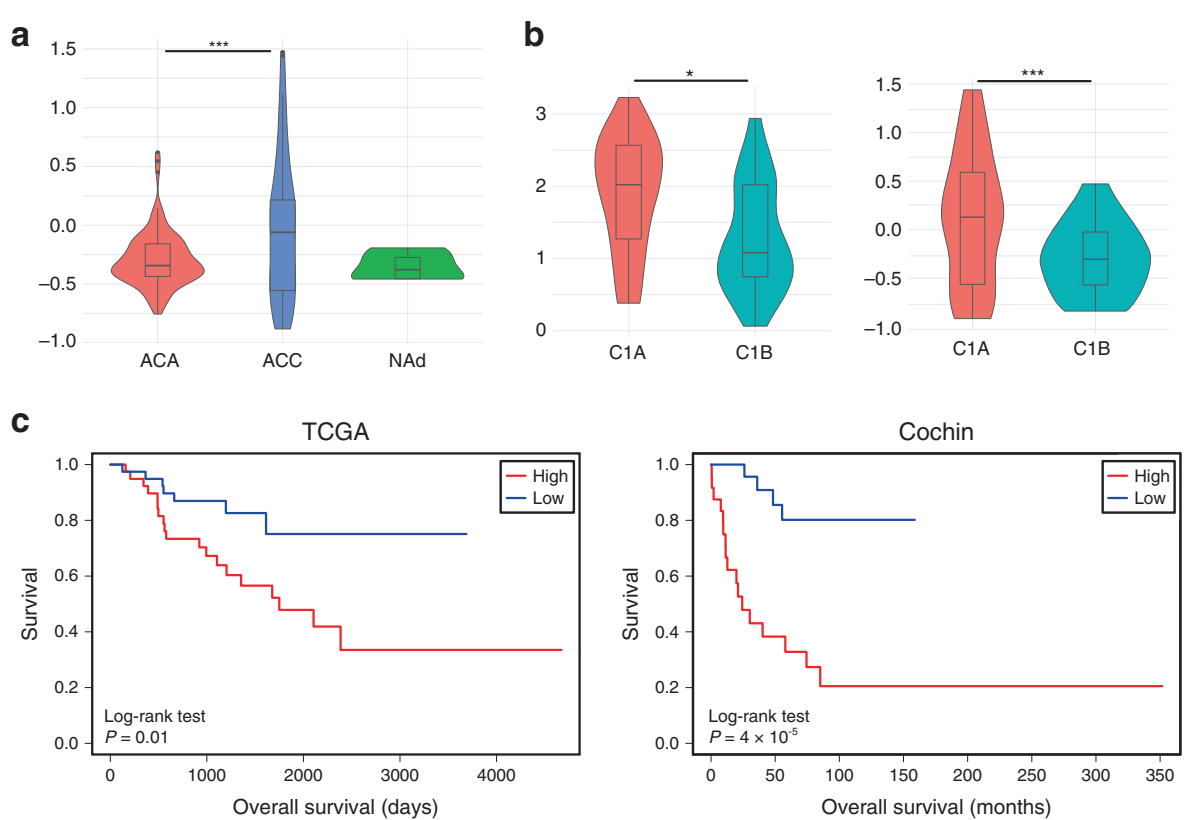

d
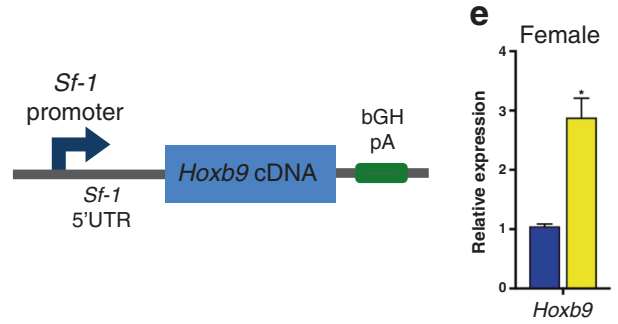

f

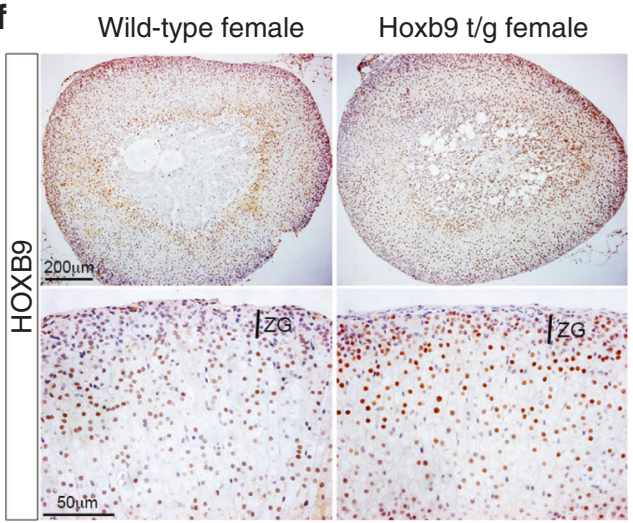

i
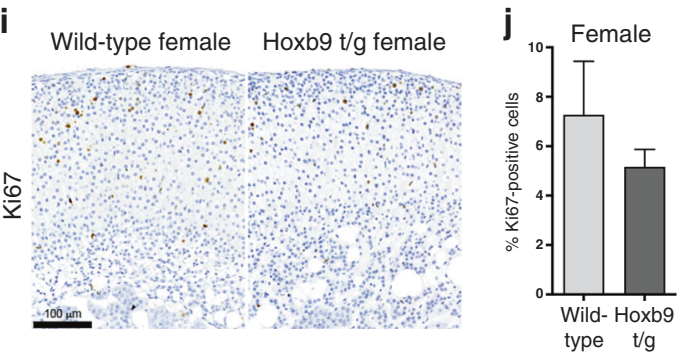

Elevated Hoxb9 cooperates with mutant Ctnnb1 during tumour formation

To investigate if HOXB9 can promote tumour formation we bred Sf-1:Hoxb9 mice with mice containing the activating conditional
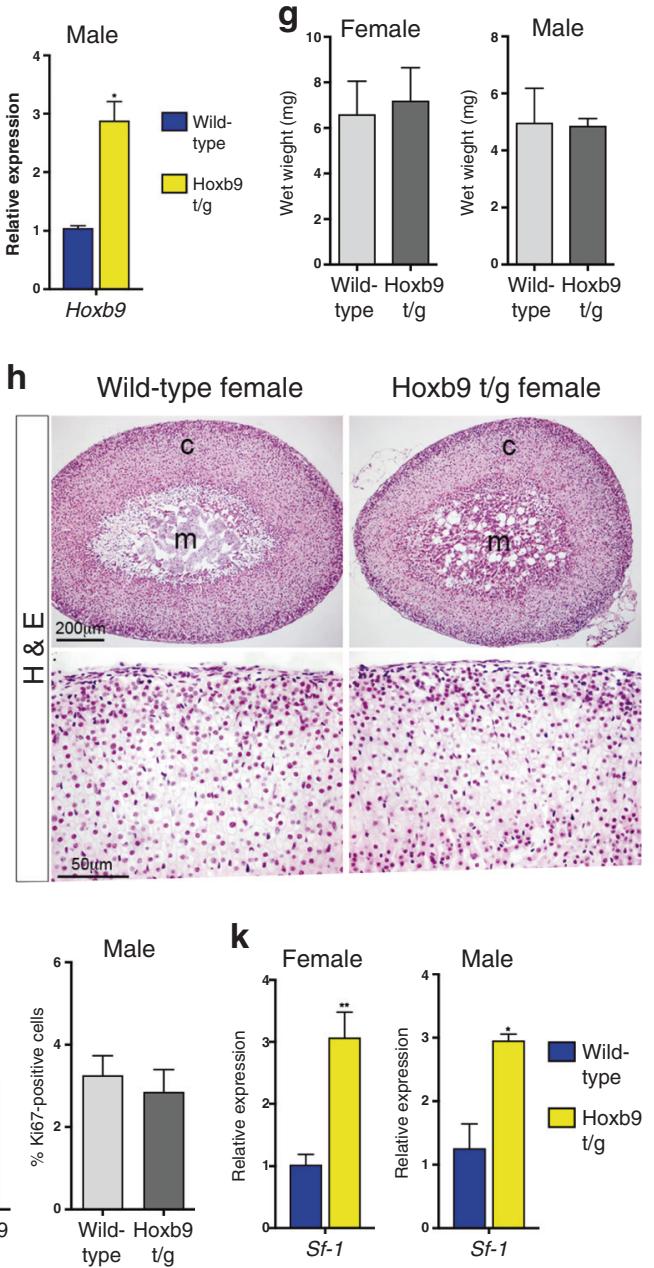

Ctnnb1 deletion allele and a construct with Cre recombinase driven by Cyp11a1-regulatory sequences (Ctnnb1 mutant mice, referred to as $A B C$ ). As expected, adrenals from Ctnnb1 mutant mice showed tumour formation characterised by increased organ 
Fig. 1 HOXB9 is associated with aggressive ACC and the generation of a transgenic mouse overexpressing Hoxb9 in adrenal cells. a HOXB9 gene expression in normal human adrenals (NAd), adrenocortical adenoma (ACA) samples, and adrenocortical carcinoma (ACC) samples from the Cochin cohort. Statistical analysis is one-way ANOVA Turkey's pairwise test, $* * * P=0.0004$. b HOXB9 expression in ACC samples from patients with aggressive C1A or indolent C1B-type disease from TCGA and Cochin cohorts. Statistical analysis is a Wilcoxon test, ${ }^{* * *} P=0.00053,{ }^{*} P=0.04$. c Kaplan-Meier survival curves for ACC patients from the TCGA and Cochin cohorts that had either high or low HOXB9 expression. d Schematic of the Sf-1:Hoxb9 transgenic construct used to increase Hoxb9 expression in adrenal glands. bGH pA is a bovine growth hormone polyA sequence. e qRT-PCR of Hoxb9 on wild-type and Hoxb9 t/g adrenal glands. The data represent mean \pm SD from three biological repeats. f HOXB9 immunohistochemistry on sections of female wild-type and Hoxb9 t/g adrenal glands. ZG is zona glomerulosa. $g$ Wet weights of male and female adrenal glands from wild-type and Hoxb9 t/g animals. The data represent mean \pm SD from four samples. h Haematoxylin and eosin (H\&E) stain on sections from wild-type and Hoxb9 t/g adrenal glands. $\mathrm{c}$ is the cortex, $\mathrm{m}$ is the medulla. i Ki67 immunohistochemistry on sections of wild-type and Hoxb9 t/g female adrenal glands. $\mathbf{j}$ Bar chart of the percentage of Ki67-positive cells in wild-type and Hoxb9 t/g male and female adrenal glands. The data represent the mean \pm SD from three biological repeats. $\mathbf{k}$ qRT-PCR of Sf-1 on wild-type and Hoxb9 $\mathrm{t} / \mathrm{g}$ adrenal glands. The data represent the mean \pm SD from three biological repeats. Student's $t$ test, ${ }^{* *} P<0.01,{ }^{*} P<$ 0.05. Hoxb9 t/g indicates Sf-1:Hoxb9 transgenic.

size, which was larger in the female (Figs. $1 \mathrm{~g}$ and $3 \mathrm{~b}$ ). These tumours had a lack of zonal structure, loss of medullar cells and high expression of the ZG marker Dab2 throughout the tumour (Supplementary Fig. S3A, B). Six-month-old Ctnnb1 mutant mice that also carried the Sf-1:Hoxb9 transgene (double-mutant mice, referred to as $A B C$; Hoxb9 $t / g$ ) showed an increase in adrenal size, which was restricted to male mice (Fig. 3a, b). Haematoxylin and eosin staining showed no obvious morphological difference between Ctnnb1 and double mutants (Fig. 3c). As expected, antibody staining for $\beta$-catenin and the WNT signalling downstream marker Lef1 in Ctnnb1 mutants showed high expression in the majority of cells (Fig. 3c). This staining pattern was unchanged in double-mutant tumours showing that elevated Hoxb9 had no major effect on this pathway (Fig. 3c). Proliferation, as measured by Ki67 staining, was higher in 6-month-old double-mutant male mice, with no changes in apoptosis, as measured by Caspase 3 staining (Fig. $3 \mathrm{~d}-\mathrm{g}$ ). No signs of invasive disease were observed in double-mutant animals (Fig. 3c, arrows). Adrenal tumours from double-mutant mice expressed higher levels of Hoxb9 mRNA and protein than single Ctnnb1 mutants, confirming expression of the transgene (Fig. 3h, i). As Sf-1:Hoxb9 adrenals showed an increase in $S f-1$ expression, we investigated the levels of this gene in the tumours. qRT-PCR analysis showed that $S f-1$ transcript was higher in both female and male double-mutant adrenal tumours relative to Ctnnb1 mutants, but there was no difference in the levels of SF1 protein expression between the genotypes (Supplementary Fig. S3C, D).

To investigate the pathways activated in the double mutants we performed RNA-seq on RNA derived from adrenal tumours from 3month-old Ctnnb1 and double-mutant female and male mutant mice. Comparative analysis identified differentially expressed genes in the tumours of double-mutant mice compared to Ctnnb1 mutants, with a higher number in males (533 genes altered, Benjamini-Hochberg adjusted $P<0.1$ ) than in the same comparison in females (66 genes altered, Benjamini-Hochberg adjusted $P<0.1$ ) (Fig. 4a and Supplementary Tables S2-S5). For both sexes, genes were differentially up- and downregulated (males 232 genes up, 322 genes down; females 47 up, 19 genes down), consistent with evidence suggesting that HOX proteins can act as transcriptional activators and repressors.

Comparative analysis of the differentially expressed genes between double mutants and Ctnnb1 mutant female and male tumours showed very few common genes altered in both sexes (three genes upregulated, and six genes downregulated in both females and males) (Fig. 4b). Validating our qRT-PCR result, Sf-1 was increased in double mutants of both sexes. Interestingly, many genes that were differential between male double mutants and Ctnnb1 mutants were shared with those that were different between male and female Ctnnb1 mutant animals (52 upregulated genes and 30 downregulated genes) (Fig. 4c). These data suggest that Hoxb9 acts to promote tumorigenic pathways that are repressed in the male adrenal.
Pathway analysis of the differentially expressed genes using the STRING protein-protein interaction database identified angiotensin signalling enriched in double-mutant male tumours compared to Ctnnb1 mutant tumours, including Agtr1b, Egr1, Nr4a1 and members of the Fos/Jun family Fos, Fosb and Junb (Fig. 4d and Supplementary Table S4). ${ }^{34}$ Interestingly, Cyp 11 b2 expression, a target of this pathway, was not changed in these tumours. qRTPCR was used to validate these results for the Fos/Jun family, and antibody staining showed widespread expression of Fosb in double-mutant tumours (Fig. 4e, f). Gene set enrichment analysis of the RNA-seq data from male Ctnnb1 and double-mutant tumours revealed enrichment in cell cycle genes in tumours with elevated Hoxb9, consistent with the increase in proliferation in double mutants (Fig. $4 \mathrm{~g}$ and Supplementary Table S6). These data were validated using qRT-PCR for Cdk1, Ccnb1, Ccnb2, Ccne1 and Knstrn, which showed an increase in these genes in male double mutants but not in females (Fig. 4h). We next checked if the pathways we identified were also altered in female Ctnnb1 mutant tumours, compared to males of the same genotype, as these tumours shared a large number of altered genes with male double mutants (Fig. 4C). We found 15 cell cycle regulatory genes (E2F targets or G2M checkpoint Hallmark genes) upregulated in female Ctnnb1 tumours compared to Ctnnb1 male tumours, including Ccne1 and Cdk1 (Fig. 4i). These data suggest a core set of cell cycle genes that are elevated in tumours with high Hoxb9 are also expressed at high levels in female Ctnnb1 tumours, compared to males.

\section{HOX factors as potential drug targets in ACC}

Our transgenic mouse data indicates that high Hoxb9 expression can promote cell proliferation within a tumour context. We next wanted to determine if $H O X B 9$ can promote proliferation in human ACC cells. Consistent with our mouse studies, overexpression of HOXB9 in the human adrenal cortical tumour cell line H295R led to a small but significant increase in cell number (Supplementary Fig. S4A, B, C). To determine if HOXB9 expression correlates with proliferation in human samples we analysed its expression in two ACC datasets, TCGA and Cochin, with the proliferation markers MKI67, CCNE1 and an established proliferation gene signature (Wassef et al. ${ }^{32}$ ) (Fig. 5a). We found a significant correlation of HOXB9 with MKI67 and the proliferation signature in the Cochin ACC dataset but not TCGA. To investigate if other HOX genes are implicated in proliferation in human ACC we performed these correlations with all members of the HOX gene family. Several HOX genes showed a significant correlation with all proliferation markers in both datasets, including $\mathrm{HOXC9}$, HOXC10, HOXC11, HOXC13 and HOXD13 (MKI67 P<0.002, proliferation gene signature $P<0.001$, CCND1 $P<0.05$ ) (Fig. 5b, Supplementary Fig. S5, Supplementary Tables S7 and S8). An analysis of the correlation of HOX gene expression with the expression of all other HOX members in the TCGA ACC dataset showed HOXB9 expression significantly correlated with the 

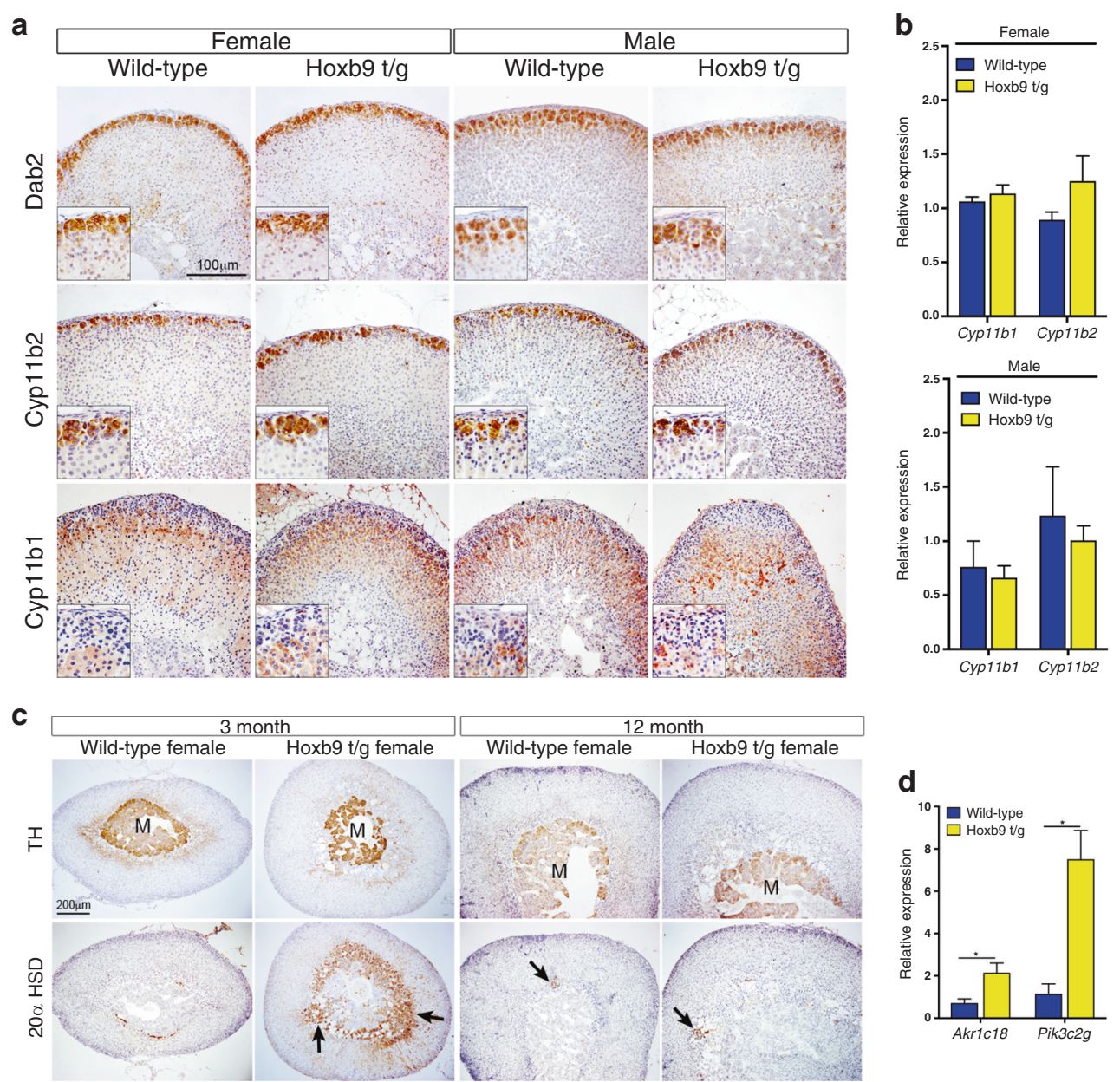

Fig. 2 Hoxb9 transgenic animals have a larger X zone. a Dab2, Cyp11b2 and Cyp11b1 immunohistochemistry on sections from wild-type and Hoxb9 t/g male and female adrenal glands. b qRT-PCR of Cyp11b1 and Cyp11b2 on wild-type and Hoxb9 t/g adrenal glands. The data represent the mean \pm SD from three biological repeats. c Tyrosine Hydroxylase (TH) and 20 $-\mathrm{HSD}$ immunohistochemistry on sections from wild-type and Hoxb9 t/g 3- and 12-month-old female adrenal glands. d qRT-PCR of Akr1c18 and Pik3c2g on female wild-type and Hoxb9 t/g adrenal glands. The data represent the mean \pm SD from three biological repeats. $\mathrm{m}$ is the medulla, arrows indicate positive cells. Student's $t$ test, ${ }^{*} P<0.05$. Hoxb9 t/g indicates Sf-1:Hoxb9 transgenics.

expression of these HOX genes (HOXC9 $r=0.377 ; P=0.000604$, HOXC10 $r=0.474 ; P=9.81^{-06}, H O X C 11 \quad r=0.427 ; P=8.60^{-05}$ HOXC13 $r=0.455 ; P=1.16^{-06}$ and HOXD13 $r=0.453 ; P=2.69^{-05}$ ) (Supplementary Tables S7, S8 and Supplementary Fig. S6).

To establish if these genes were implicated in disease progression, we performed correlations between HOX gene expression and ACC C1A versus $\mathrm{C} 1 \mathrm{~B}$ status (Fig. $5 \mathrm{C}$ and Supplementary Fig. S7), and ACC versus ACA and NAd (Fig. $5 \mathrm{~d}$ ), and found that higher levels of HOX genes correlate with ACC and aggressive disease. Analysis of overall and disease-free survival between ACC patients with high and low HOX gene expression showed a correlation between high HOX levels and poor prognosis (Fig. 5e, Supplementary Figs. S8 and S9). These data argue that HOX genes can be drivers of aggressive ACC disease.

To investigate if adrenal tumour growth is dependent on HOX genes, we performed siRNA knockdown studies of HOX genes expressed in H295R cells. ${ }^{13}$ Knockdown of HOXA11, but not HOXA10 or HOXA13, led to reduced growth of H295R cells, supporting a role of HOX genes in promoting adrenal tumour cell proliferation (Fig. 6a, b). Analysis of HOXA11 paralogues after HOXA11 knockdown showed a modest reduction in HOXC11 expression, while HOXD11 expression was not detected in H295R cells in control or siRNA-treated samples (Fig. S4D). Our HOX gene expression correlation analysis showed a strong correlation of
HOX genes within clusters, including HOXA10 with HOXA11 and HOXA13, and weaker associations with their paralogues (Supplementary Tables S7, S8 and Supplementary Fig. S6). Analysis of HOXA11 expression in the TCGA ACC dataset showed that it significantly correlated with Ki67 expression $(P=0.0023, r=0.337)$ the proliferation gene signature $(P=0.00053, r=0.381)$ and CCNE1 ( $P=0.0010, r=0.363$ ) expression in these tumours.

As we have shown that several HOX genes correlate with proliferation markers and aggressive disease in ACC we chose to target $P B X 1$, a transcription factor that cooperates with $\mathrm{HOX}$ proteins to regulate target gene expression and has been implicated in adrenal development and function. ${ }^{15}$ siRNA knockdown of PBX1 in H295R cells led to reduced levels of expression and to a marked reduction in cell proliferation (Fig. 6C, d). H295R cells harbour a CTNNB1-activating mutation, and our qRT-PCR studies showed that $P B X 1$ knockdown had no effect on WNT signalling, as measured by the expression levels of downstream targets AXIN2 and LEF1 (Supplementary Fig. S4E). In order to further investigate if HOX factors can act as drug targets in ACC, we used a developed antagonist peptide, which interferes with the interaction between HOX and PBX proteins ( $\mathrm{HTLO01}{ }^{30}$ ). Our drug response studies showed that $\mathrm{H} 295 \mathrm{R}$ cell growth was highly inhibited by HTL001 but not by a control peptide (CXR9) (Fig. 6e). The IC ${ }_{50}$ of HTL001 in H295R cells was lower than that of another 


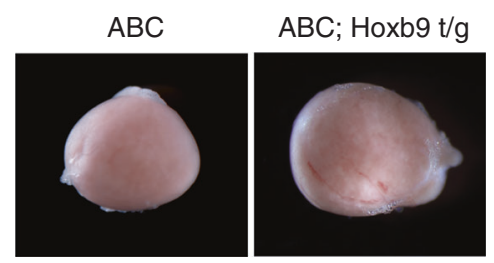

b

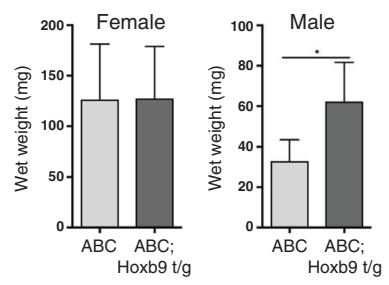

C

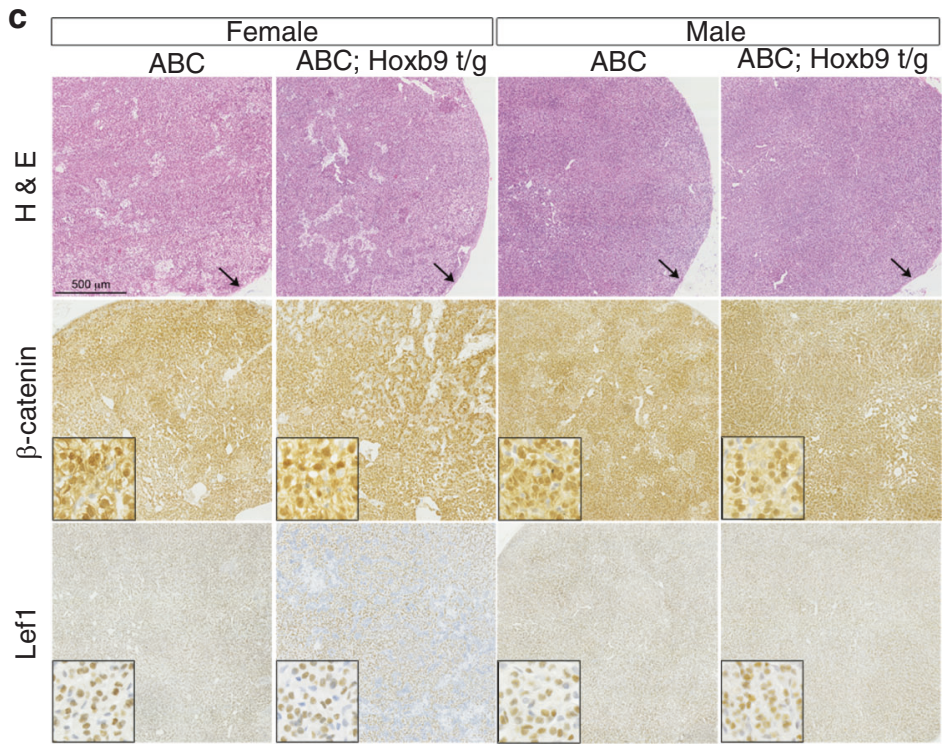

d
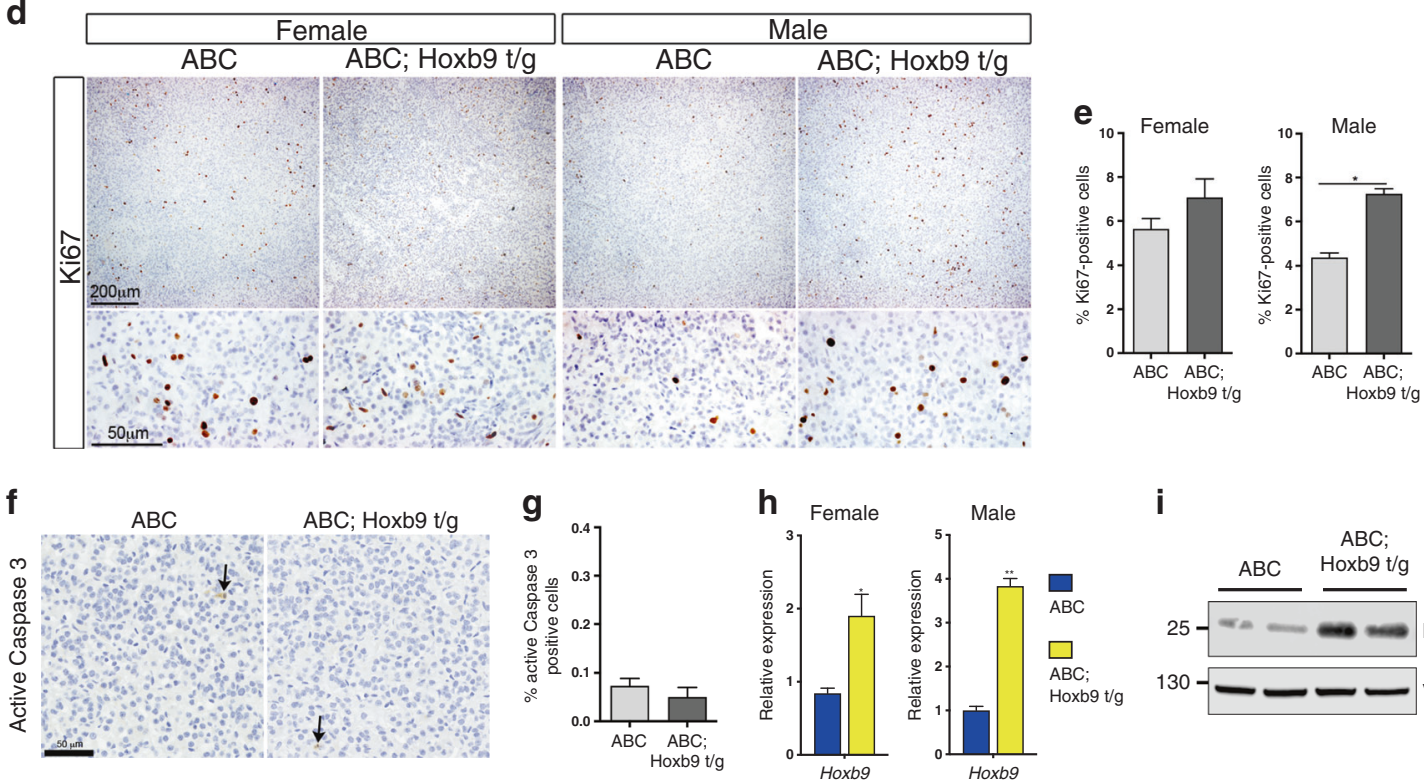

i

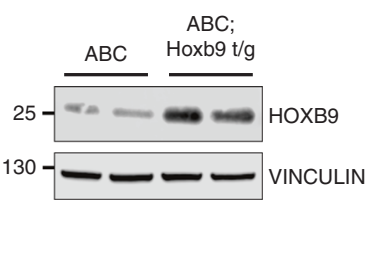

Fig. 3 Hoxb9 cooperates with Ctnnb1 during tumour formation. a Bright-field images of male $A B C$ and $A B C ;$ Hoxb9 t/g adrenal glands. b Wet weight of 6-month-old female and male $A B C$ and $A B C ;$ Hoxb9 t/g adrenal glands. The data represent the mean $\pm S D$ from four tumours. $c$ $\mathrm{H} \& \mathrm{E}, \beta$-catenin and Lef1 immunohistochemistry on sections from $A B C$ and $A B C ;$ Hoxb9 t/g adrenal tumours. Inset shows high-power magnification. Arrow indicates capsule. d Ki67 immunohistochemistry on sections from 6-month-old $A B C$ and $A B C$; Hoxb9 t/g adrenal tumours. e Bar chart of the percentage of Ki67-positive cells in ABC and ABC; Hoxb9 t/g adrenals. The data represent the mean \pm SD from three biological repeats. f Active Caspase 3 immunohistochemistry on sections from $A B C$ and $A B C ;$ Hoxb9 t/g male tumours. Arrows indicate positive cells. $\mathbf{g}$ Bar chart of the percentage of Caspase 3-positive cells in $A B C$ and $A B C ; \mathrm{Hoxb} 9 \mathrm{t} / \mathrm{g}$ male adrenals. The data represent the mean \pm SD from three biological repeats. $\mathbf{h}$ qRT-PCR of Hoxb9 on $A B C$ and $A B C$; Hoxb9 t/g adrenal tumours. The data represent mean \pm SD from three biological repeats. $i$ Western blot analysis of Hoxb9 on $A B C$ and $A B C$; Hoxb9 t/g adrenal tumours from female animals. Adrenals from two animals of each genotype are shown. Vinculin is used as a loading control. ABC indicates Ctnnb1 mutant tumours, ABC; Hoxb9 t/g indicates double-mutant tumours. Student's $t$ test, ${ }^{* *} P<0.01,{ }^{*} P<0.05$.

responsive cell line, the prostate cancer line PC3 (5.54 $\mu \mathrm{M}$ versus $30.11 \mu \mathrm{M})$. To investigate if mouse adrenal tumours were also sensitive, we analysed three models: two cell lines derived from adrenal tumours driven by SV40 T antigen, ATC1 (containing an activating Ctnnb1 mutation ${ }^{35}$ ) and ATC7; and primary cells derived from adrenal tissue from the Ctnnb1 mutant mice described above. These cells were treated with the antagonist HOX-PBX peptide and found to be responsive, with ATC7 cells being the most sensitive (IC50 23.11 $\mu \mathrm{M}$ ) (Fig. 6e). Cell death assays on H295R cells confirmed an increase in apoptosis in cells treated with the antagonist (Fig. 6f).

\section{DISCUSSION}

This study investigates the roles of HOX genes in ACC and their potential as drug targets in this disease. HOX genes have been implicated in cancer, most notably in haematological malignancies. ${ }^{36}$ In many cases, elevated expression correlates with poor prognosis, which is what we observe for HOXB9 and other HOX genes in ACC. Our data show that overexpression of Hoxb9 in the adrenal leads to an increase in foetal derived X-zone cells, but does not promote hyperplasia or neoplasia. However, in combination with an activated Ctnnb1 mutation, high levels of Hoxb9 lead to an increase in proliferation. This data suggests that 


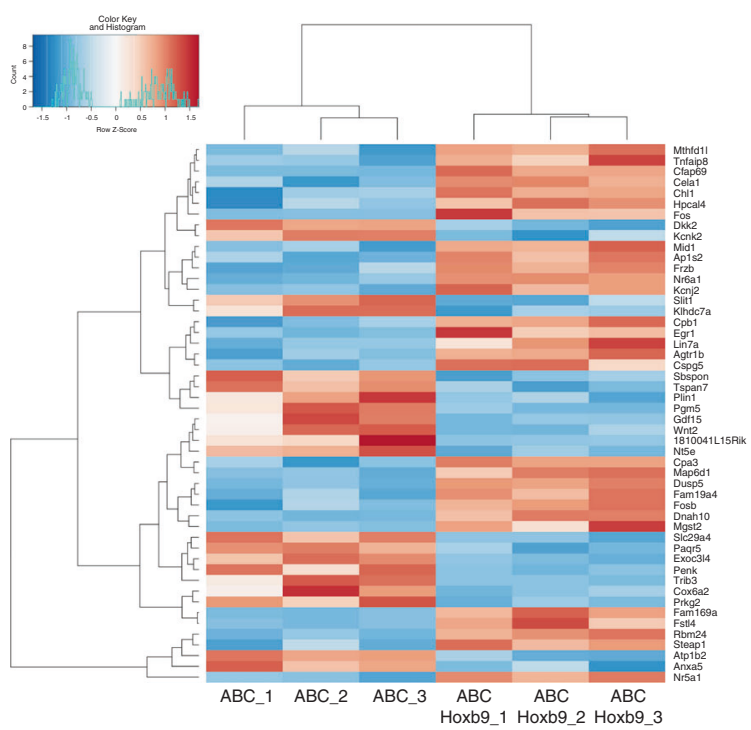

b

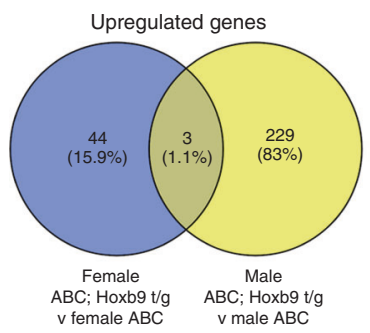

C Upregulated genes

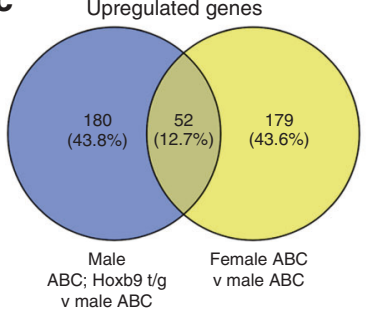

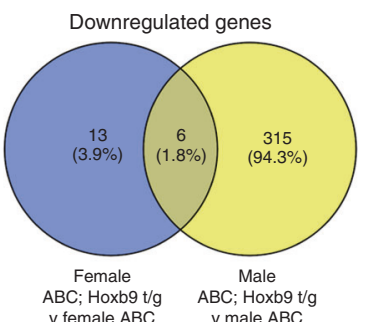

$\checkmark$ f $\quad$,

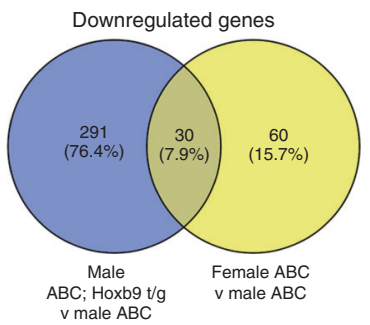

d

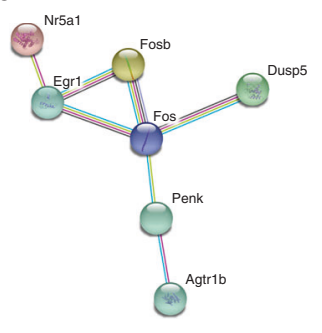

e

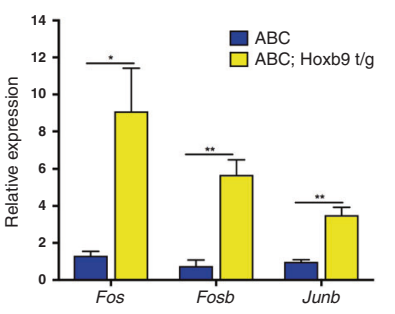

f

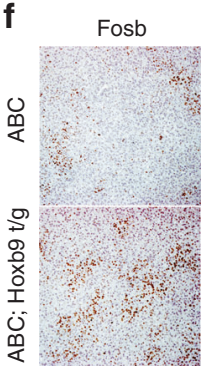

g

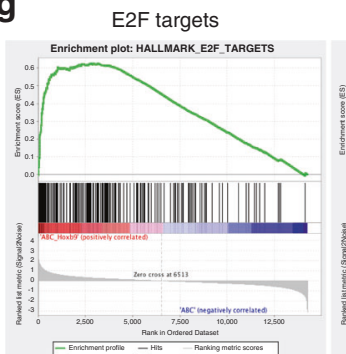

G2M checkpoint

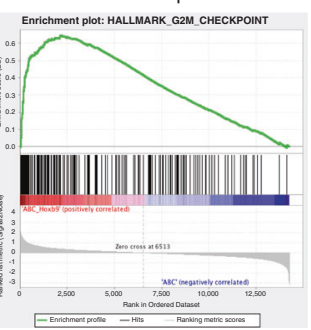

h

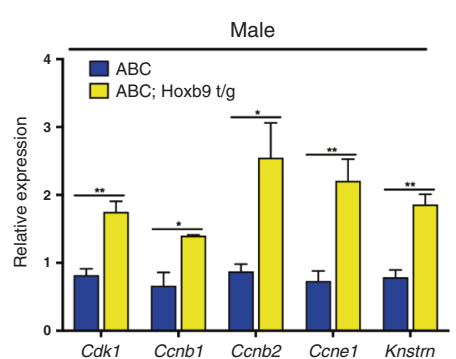

Fig. 4 Elevated HOX expression leads to an increase in cell cycle gene expression. a Heatmap of the top 50 differentially expressed genes identified from RNA-seq data of male $A B C ; H o x b 9 t / g$ adrenal tumours compared to male $A B C$ adrenal tumours. $\mathbf{b}$ Comparison of differentially expressed genes in male and female $A B C$; Hoxb9 t/g adrenal tumours compared to $A B C$ adrenal tumours. c Comparison of differentially expressed genes in male $A B C ;$ Hoxb9 $t / g$ tumours compared in male $A B C$ tumours to the genes differentially expressed genes in female $A B C$ tumours compared to male ABC tumours. d STRING database network of differentially expressed genes in male ABC; Hoxb9 t/g tumours compared to male $A B C$ tumours identifies angiotensin signalling. e qRT-PCR of Fos, Fosb and Junb in male $A B C$ and $A B C ;$ Hoxb9 t/g tumours. The data represent mean \pm SD from three biological repeats. $f$ IHC of Fosb in 3-month-old male $A B C ;$ Hoxb9 t/g and $A B C$ adrenal tumours. g GSEA of RNA-seq data from male $A B C ;$ Hoxb9 t/g and male $A B C$ adrenal tumours. E2F targets normalised enrichment score (NES) $=1.57$, FDR $q=0.069, \mathrm{G} 2 \mathrm{M}$ checkpoint NES $=1.70, \mathrm{FDR} q=0.043$. h qRT-PCR of Cdk1, Ccnb1, Ccnb2, Ccne 1 and Knstrn in female and male ABC and ABC; Hoxb9 t/g adrenal tumours. The data represent mean \pm SD from three biological repeats. i Cell cycle genes upregulated in male ABC; Hoxb9 t/ $g$ tumours and female $A B C$ tumours (compared to $A B C$ males). Student's $t$ test, ${ }^{* *} P<0.01,{ }^{*} P<0.05$. ABC indicates Ctnnb1 mutant tumours, $A B C$; Hoxb9 t/g indicates double-mutant tumours.

HOX genes do not initiate neoplasia but can promote aggressive disease with an increase in cell cycle-dependent genes, such as Cyclin E1. Our studies also show that adrenal tumour cells are dependent on the HOX-PBX interaction for growth and that therefore these factors have the potential to be drug targets in ACC.

ACC has a sex bias, with females being more at risk than males. ${ }^{37}$ Consistent with this, mice with Ctnnb1-activating mutations in the adrenal show a more aggressive and earlier phenotype in females, which we also observe in our Ctnnb1 mutant mice. Recent data have shown a sex-specific difference in cell proliferation and renewal in the adrenal cortex, with females showing higher turnover and using an additional stem/progenitor compartment found in the capsule. ${ }^{38}$ This difference was driven by testicular androgens that repressed these processes in the male. Our data is consistent with Hoxb9 promoting the proliferative pathways that are repressed in the male. Our RNAseq analysis did not show changes in classic androgen receptor targets, such as Srd5a2, however, we did observe an increase in Frzb expression in double-mutant adrenals, which has been shown to be higher in female adrenals and upregulated in adrenals of castrated mice. ${ }^{39}$ 

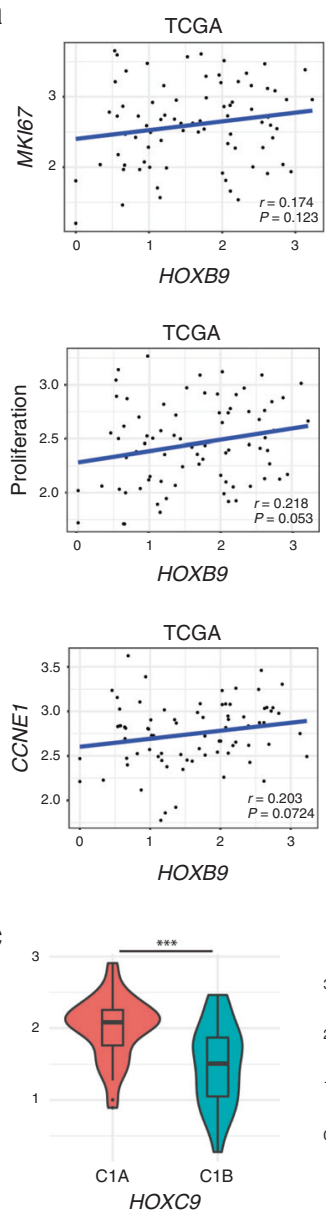

d

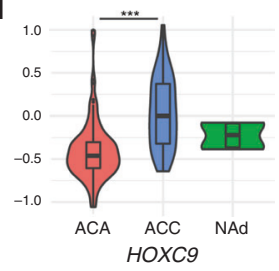

e

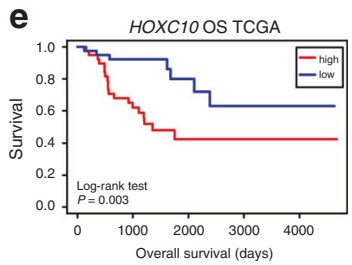

HOXD13OS TCGA

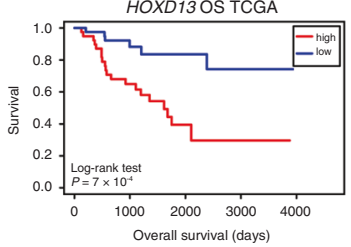

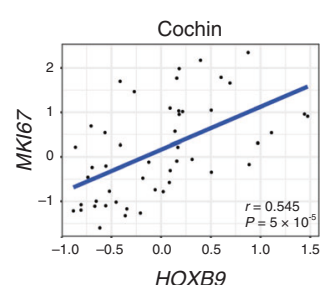
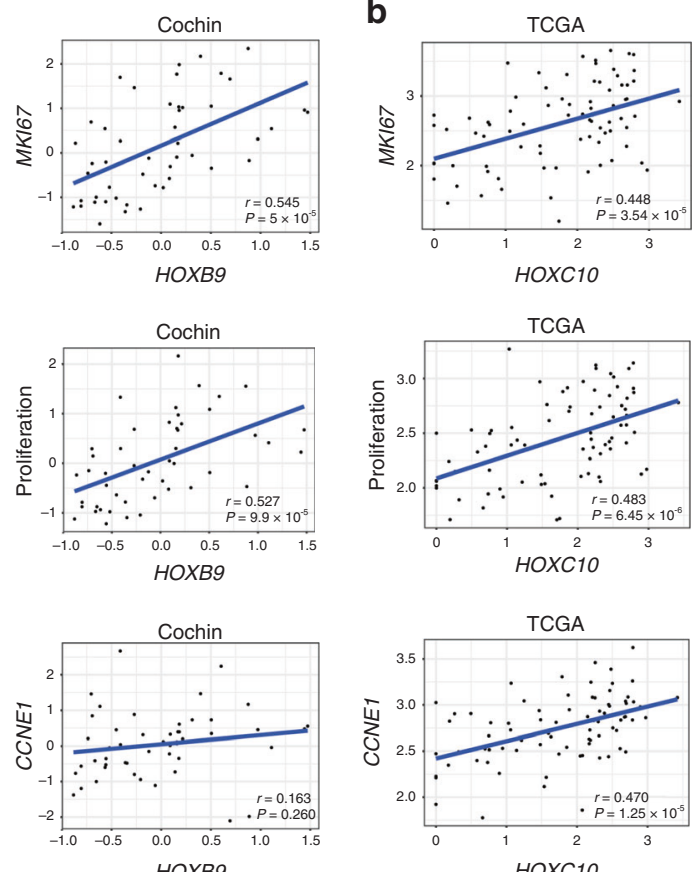
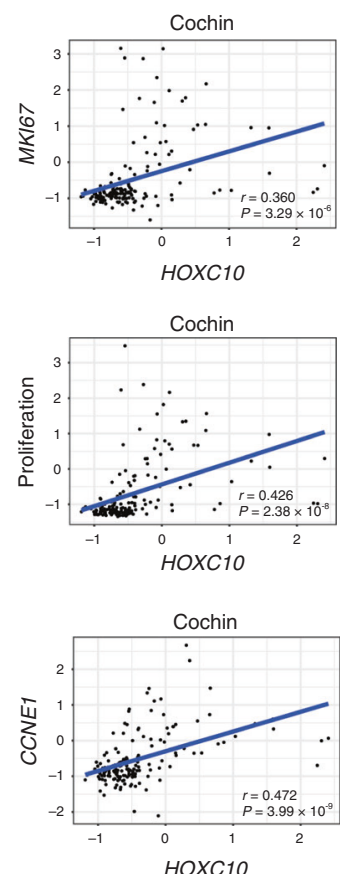
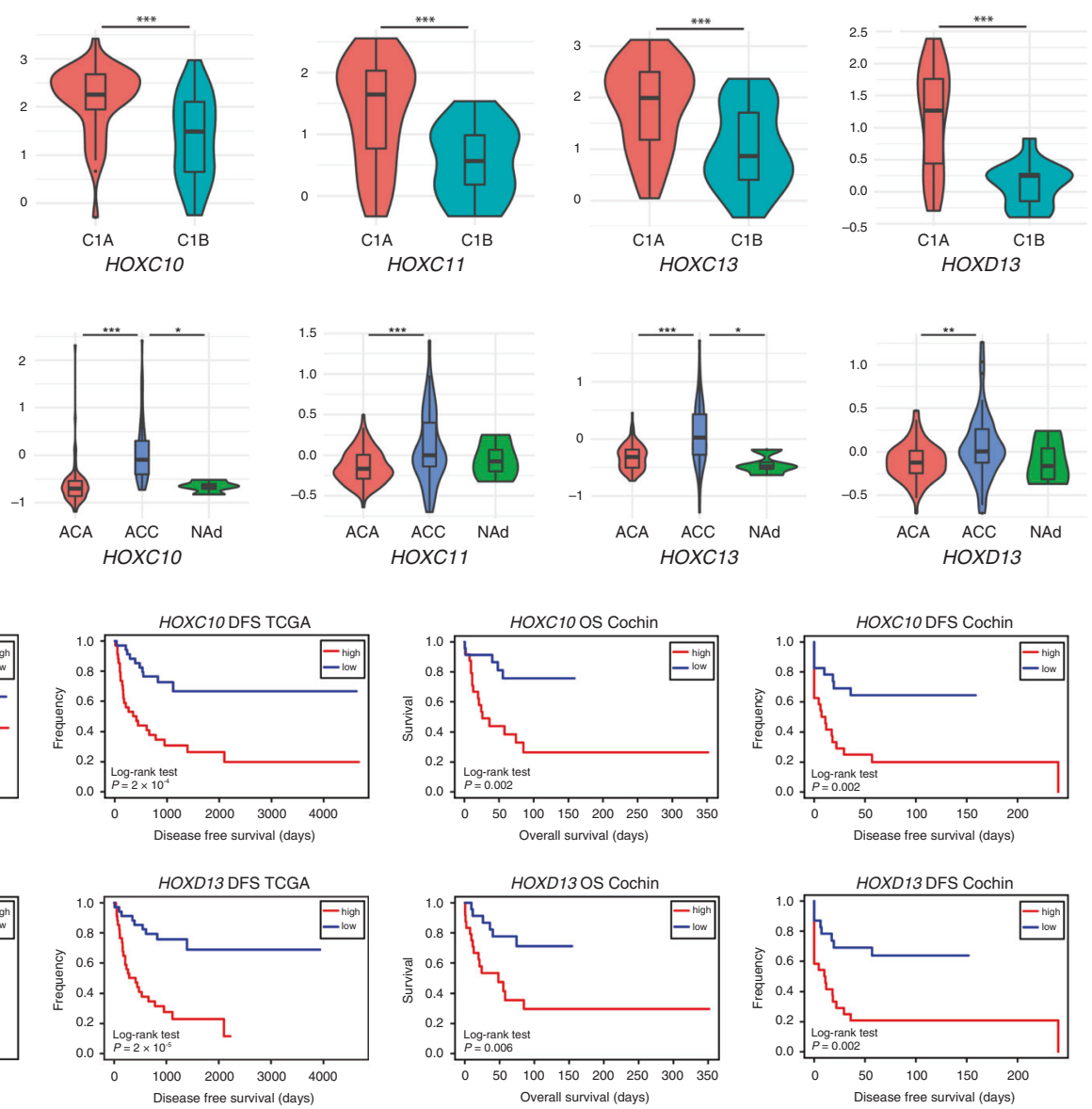

Fig. 5 HOX gene expression is associated with aggressive ACC. a Correlation of HOXB9 expression with MKI67, a proliferation gene signature, and CCNE1 in ACC patient samples from the TCGA and Cochin cohorts. b Correlation of HOXC10 expression with MKI67, a proliferation gene signature, and CCNE1 in ACC patient samples from the TCGA and Cochin cohorts. c HOX gene expression in ACC samples from patients with aggressive C1A or indolent C1B-type disease from the TCGA cohort. Statistical analysis a Wilcoxon test, ${ }^{* * *} P<0.001$. d $H O X$ gene expression in normal human adrenals (NAd), adrenocortical adenoma (ACA) samples, and adrenocortical carcinoma (ACC) samples from the Cochin cohort. Statistical analysis is one-way ANOVA Turkey's pairwise test, ${ }^{* * *} P<0.001,{ }^{* *} P<0.01,{ }^{*} P<0.05$. e Kaplan-Meier survival curves for ACC patients from the TCGA and Cochin cohorts that had either high or low HOXC10 or HOXD13 expression. 
a

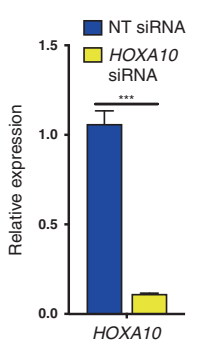

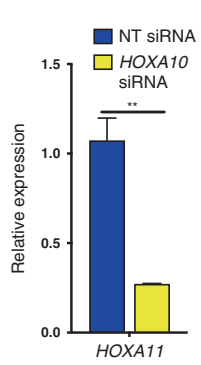
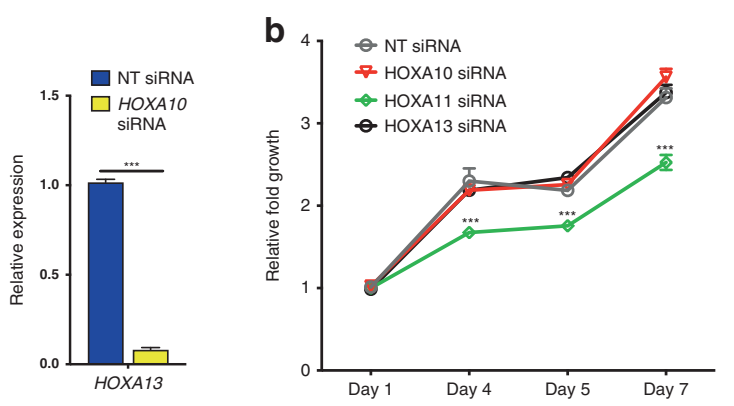

C
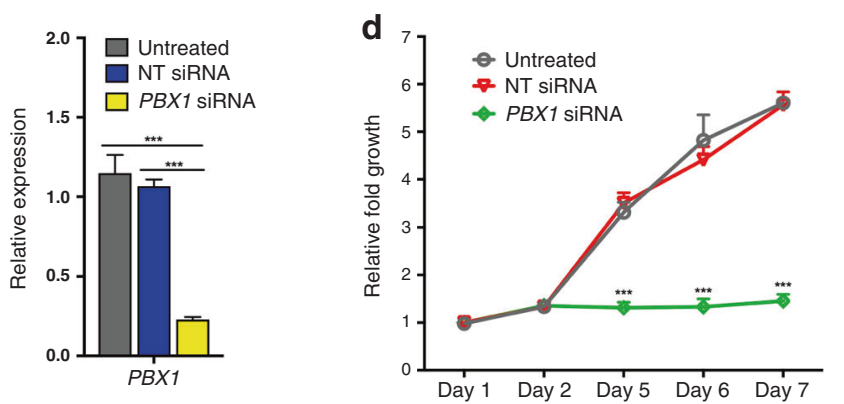

e

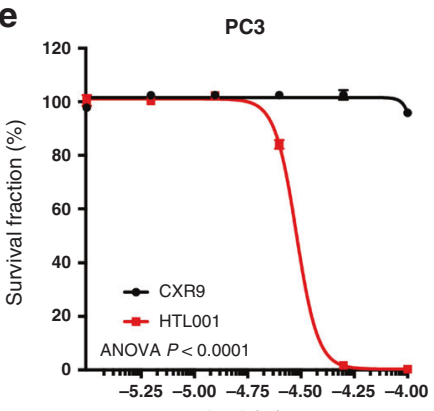

[log] $(\mathrm{M})$
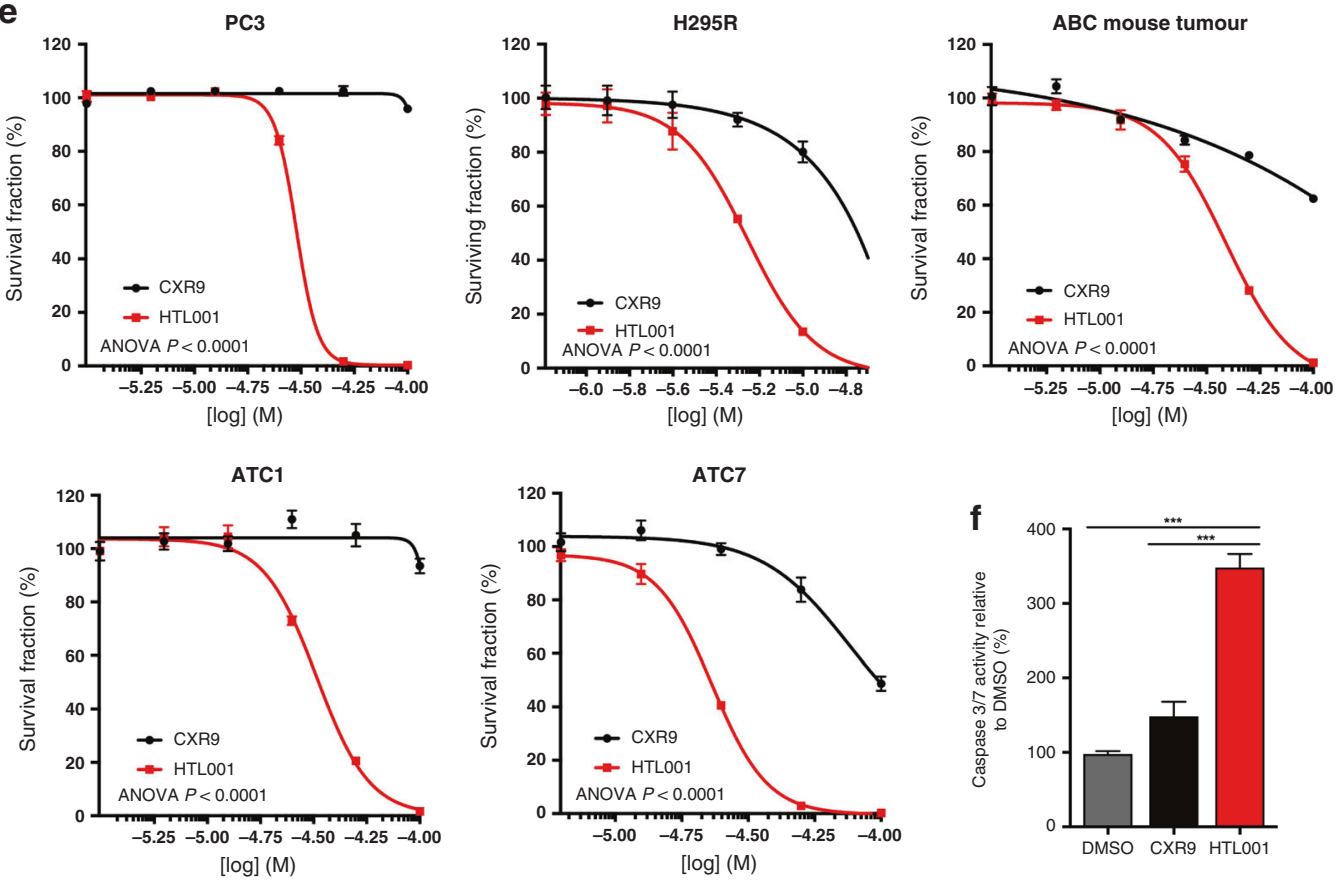

Fig. 6 ACC cells are sensitive to inhibition of HOX-PBX function. a qRT-PCR of HOXA10, HOXA11 or HOXA13 in H295R cells treated with a non-targeting (NT) siRNA or a siRNA targeting HOXA10, HOXA11 or HOXA13. The data represent the mean \pm SD from three biological repeats. $\mathbf{b}$ Growth curve of H295R cells treated with a NT siRNA or a siRNA targeting HOXA10, HOXA11 or HOXA13. c qRT-PCR of PBX1 in untreated H295R cells, cells treated with a NT siRNA, or a siRNA targeting PBX1. The data represent the mean \pm SD from three biological repeats. d Growth curve of untreated H295R cells, cells treated with a NT siRNA, or a siRNA targeting PBX1. e Survival fraction of PC3, H295R, ABC (Ctnnb1 mutant) mouse adrenal cells, ATC1 and ATC7 cells treated with HTL001 or CXR9. Cell viability was measured using Cell TitreGlo. f Caspase 3/7 activity for H295R cells treated with $5 \mu \mathrm{M}$ HTL001 (IC50), DMSO or $5 \mu \mathrm{M}$ CXR9 for $24 \mathrm{~h}$. The data represent the mean \pm SD from three biological repeats. One-way ANOVA, ${ }^{* *} P<0.01,{ }^{* * *} P<0.001$.

Overexpression of Hoxb9 led to an increase in $\mathrm{Sf}-1$ levels both in a normal and neoplastic context. HOX genes have been implicated in the regulation of $S f-1$ transcription in the developing adrenal. ${ }^{14}$ Transgenic mice with increased foetal $S f-1$ expression showed extra-adrenal formation and mice with a Sf-1 sumoylation mutation have a persistent $X$ zone, suggesting that the bigger foetal derived $X$ zone seen in the adrenal of the transgenic Sf-1:
Hoxb9 mice could be due to the higher $S f-1$ levels. ${ }^{40,41}$ Whether this pathway is active in adrenal tumours is unclear as we did not observe an increase in $\mathrm{Sf}-1$ protein in the adrenals of doublemutant mice.

Our data show an increase in cell cycle markers in doublemutant male tumours including Cone1, a gene commonly amplified in ACC and thought to be a disease driver. Both HOX 
genes and SF-1 have been implicated in the regulation of CCNE1 expression. ${ }^{42,43}$ A surprising result was the observation of an increase in the expression of the FOS/JUN/EGR1 pathway without an increase in Cyp $11 b 2$ expression. ${ }^{34}$ Analysis of Fosb staining in normal adrenals did show its association with the ZG, the site of angiotensin signalling, but was also found in a few capsule cells (Supplementary Fig. S10). This suggests that the FOS/JUN family can be activated in non-steroidogenic cells of the adrenal. Whether its function in these cells is associated with cell proliferation is not known.

WNT signalling has been implicated in adrenal development and differentiation, as well as homoeostasis and neoplasia in the adult. The double-mutant animals in our study do not show major changes in differentiation, such as Cyp11b1 or Cyp11b2, or stem cell markers, such as Shh and Gli1, when compared to the Ctnnb1 mutants. The major difference we observe is an increase in proliferation in double-mutant male adrenals. Mice with Znrf3 mutations show an increase in proliferation of the ZF, which is thought to be due to moderate rather than high WNT signalling activation. ${ }^{44}$ We do not observe any major changes in WNT target genes in the neoplastic tissues, suggesting that HOX genes do not act to modulate this pathway in adrenal disease.

Our analysis of HOX gene expression revealed various members of the family had increased levels in human ACC, some of which correlated with increased proliferation markers. Our experiments in H295R cells identified a need for HOXA11, but not for other HOX expressed genes, HOXA10 and HOXA13, for survival. This variety prompted us to propose a more general HOX-based therapy for patient treatment as different tumours may have specific HOX gene combinations driving disease progression. Studies have shown that functional redundancy in organ formation can be observed in paralogous members of the HOX gene family. ${ }^{45}$ Our expression studies in ACC samples comparing members within the HOX family showed that correlations were higher in genes of the same cluster rather than their related paralogues. Consistent with this, analysis of the HOXA11 paralogues in H295R cells with HOXA11 knockdown only showed a mild reduction in HOXC11 expression. HOX transcript antisense intergenic RNA (HOTAIR) has been implicated in ACC with high expression correlating with worse disease outcome. ${ }^{46}$ HOTAIR has been proposed to regulate the expression of many genes through its interaction with polycomb repressive complex 2 . How this LncRNA interacts with HOX genes in ACC is not known.

Our studies show that adrenal tumour cells are dependent on HOX genes for their growth, although we were not able to predict which member of the family is the most relevant to patients. As a way to overcome this issue, we determined a dependency of adrenal tumour cells on the interaction between HOX and PBX factors for growth. This makes inhibiting this interaction a promising therapy option for ACC. Peptide-based therapeutics are being used in cancer treatment and a variant of HTL001 is being developed for clinical trials currently, as in vivo studies in mice showed growth inhibition in a range of tumour types, and the drug was well-tolerated. ${ }^{47}$ We treated various adrenal tumour models with the HOX peptide inhibitor and saw an effect on cell survival. This suggests that this therapy would be effective in ACC with different genetic backgrounds.

\section{ACKNOWLEDGEMENTS}

We thank the Tumour Profiling Unit for generating the RNA-seq data. We thank Adrienn Zsippai for her experimental help. We thank Hardev Pandha for advice on the peptide inhibitors used in this study.

\section{AUTHOR CONTRIBUTIONS}

A.S., J.C.F. and P.V. conceived and designed the experiments. J.C.F. and J.R.G. performed the experiments. J.C.F, Y.R., P.V. and R.C. carried out bioinformatic analyses. Y.W., C.G-S., A.L-M. and J.B. contributed reagents or patient datasets. A.S., J. C.F. and P.V. analysed the data and wrote the paper.

\section{ADDITIONAL INFORMATION}

Ethics approval and consent to participate All animal experiments were performed in accordance with the Animal (Scientific Procedures) Act 1986 and with relevant guidelines and regulations of the Institute of Cancer Research Animal Welfare and Ethical Review Board that approved the study.

Consent to publish Not applicable.

Data availability RNA-seq data can be found in the supplementary tables.

Competing interests The authors declare no competing interests.

Funding information J.C.F. and this work was funded by an MRC grant (MR/ N009916/1). J.G. was funded by an MRC grant (G0901645/1).

Supplementary information is available for this paper at https://doi.org/10.1038/ s41416-020-01166-z.

Publisher's note Springer Nature remains neutral with regard to jurisdictional claims in published maps and institutional affiliations.

\section{REFERENCES}

1. Allolio, B. \& Fassnacht, M. Clinical review: adrenocortical carcinoma: clinical update. J. Clin. Endocrinol. Metab. 91, 2027-2037 (2006).

2. Else, T., Kim, A. C., Sabolch, A., Raymond, V. M., Kandathil, A., Caoili, E. M. et al. Adrenocortical carcinoma. Endocr. Rev. 35, 282-326 (2014).

3. Assie, G., Letouze, E., Fassnacht, M., Jouinot, A., Luscap, W., Barreau, O. et al. Integrated genomic characterization of adrenocortical carcinoma. Nat. Genet. 46, 607-612 (2014).

4. Juhlin, C. C., Goh, G., Healy, J. M., Fonseca, A. L., Scholl, U. I., Stenman, A. et al. Whole-exome sequencing characterizes the landscape of somatic mutations and copy number alterations in adrenocortical carcinoma. J. Clin. Endocrinol. Metab. 100, E493-E502 (2015).

5. Zheng, S., Cherniack, A. D., Dewal, N., Moffitt, R. A., Danilova, L., Murray, B. A. et al. Comprehensive pan-genomic characterization of adrenocortical carcinoma. Cancer Cell 30, 363 (2016).

6. Berthon, A., Sahut-Barnola, I., Lambert-Langlais, S., de Joussineau, C., DamonSoubeyrand, C., Louiset, E. et al. Constitutive beta-catenin activation induces adrenal hyperplasia and promotes adrenal cancer development. Hum. Mol. Genet. 19, 1561-1576 (2010).

7. Kim, A. C., Reuter, A. L., Zubair, M., Else, T., Serecky, K., Bingham, N. C. et al. Targeted disruption of beta-catenin in Sf1-expressing cells impairs development and maintenance of the adrenal cortex. Development 135, 2593-2602 (2008).

8. Drelon, C., Berthon, A., Sahut-Barnola, I., Mathieu, M., Dumontet, T., Rodriguez, S. et al. PKA inhibits WNT signalling in adrenal cortex zonation and prevents malignant tumour development. Nat. Commun. 7, 12751 (2016).

9. Vidal, V., Sacco, S., Rocha, A. S., da Silva, F., Panzolini, C., Dumontet, T. et al. The adrenal capsule is a signaling center controlling cell renewal and zonation through Rspo3. Genes Dev. 30, 1389-1394 (2016).

10. Kim, A., Giordano, T. J., Kuick, R., Serecky, K. \& Hammer, G. D. Wnt/betacatenin signaling in adrenocortical stem/progenitor cells: implications for adrenocortical carcinoma. Ann. Endocrinol. 70, 156 (2009).

11. Shah, N. \& Sukumar, S. The Hox genes and their roles in oncogenesis. Nat. Rev. Cancer 10, 361-371 (2010).

12. Schnabel, C. A., Selleri, L. \& Cleary, M. L. Pbx1 is essential for adrenal development and urogenital differentiation. Genesis 37, 123-130 (2003).

13. Neville, S. E., Baigent, S. M., Bicknell, A. B., Lowry, P. J. \& Gladwell, R. T. Hox gene expression in adult tissues with particular reference to the adrenal gland. Endocr. Res. 28, 669-673 (2002).

14. Zubair, M., Ishihara, S., Oka, S., Okumura, K. \& Morohashi, K. Two-step regulation of Ad4BP/SF-1 gene transcription during fetal adrenal development: initiation by a Hox-Pbx1-Prep1 complex and maintenance via autoregulation by Ad4BP/SF-1. Mol. Cell Biol. 26, 4111-4121 (2006).

15. Lichtenauer, U. D., Duchniewicz, M., Kolanczyk, M., Hoeflich, A., Hahner, S., Else, T. et al. Pre-B-cell transcription factor 1 and steroidogenic factor 1 synergistically 
regulate adrenocortical growth and steroidogenesis. Endocrinology 148, 693-704 (2007).

16. Abe, M., Hamada, J., Takahashi, O., Takahashi, Y., Tada, M., Miyamoto, M. et al. Disordered expression of HOX genes in human non-small cell lung cancer. Oncol. Rep. 15, 797-802 (2006).

17. Miao, J., Wang, Z., Provencher, H., Muir, B., Dahiya, S., Carney, E. et al. HOXB13 promotes ovarian cancer progression. Proc. Natl Acad. Sci. USA 104, 17093-17098 (2007).

18. Wu, X., Chen, H., Parker, B., Rubin, E., Zhu, T., Lee, J. S. et al. HOXB7, a homeodomain protein, is overexpressed in breast cancer and confers epithelialmesenchymal transition. Cancer Res. 66, 9527-9534 (2006).

19. Zhai, Y., Kuick, R., Nan, B., Ota, I., Weiss, S. J., Trimble, C. L. et al. Gene expression analysis of preinvasive and invasive cervical squamous cell carcinomas identifies HOXC10 as a key mediator of invasion. Cancer Res. 67, 10163-10172 (2007).

20. Miller, G. J., Miller, H. L., van Bokhoven, A., Lambert, J. R., Werahera, P. N., Schirripa, O. et al. Aberrant HOXC expression accompanies the malignant phenotype in human prostate. Cancer Res. 63, 5879-5888 (2003).

21. Jung, C., Kim, R. S., Zhang, H. J., Lee, S. J. \& Jeng, M. H. HOXB13 induces growth suppression of prostate cancer cells as a repressor of hormone-activated androgen receptor signaling. Cancer Res. 64, 9185-9192 (2004).

22. Zhang, B., Li, N. \& Zhang, H. Knockdown of homeobox B5 (HOXB5) inhibits cell proliferation, migration, and invasion in non-small cell lung cancer cells through inactivation of the Wnt/beta-catenin pathway. Oncol. Res. 26, 37-44 (2018).

23. Nguyen, D. X., Chiang, A. C., Zhang, X. H., Kim, J. Y., Kris, M. G., Ladanyi, M. et al. WNT/TCF signaling through LEF1 and HOXB9 mediates lung adenocarcinoma metastasis. Cell 138, 51-62 (2009).

24. Xiong, H., Xiao, H., Luo, C., Chen, L., Liu, X., Hu, Z. et al. GRP78 activates the Wnt/ HOXB9 pathway to promote invasion and metastasis of hepatocellular carcinoma by chaperoning LRP6. Exp. Cell Res. 383, 111493 (2019).

25. Gao, D. \& Chen, H. Q. Specific knockdown of HOXB7 inhibits cutaneous squamous cell carcinoma cell migration and invasion while inducing apoptosis via the Wnt/ beta-catenin signaling pathway. Am. J. Physiol. Cell Physiol. 315, C675-C686 (2018).

26. Guo, J., Zhang, T. \& Dou, D. Knockdown of HOXB8 inhibits tumor growth and metastasis by the inactivation of Wnt/beta-catenin signaling pathway in osteosarcoma. Eur. J. Pharm. 854, 22-27 (2019).

27. Buaas, F. W., Gardiner, J. R., Clayton, S., Val, P. \& Swain, A. In vivo evidence for the crucial role of SF1 in steroid-producing cells of the testis, ovary and adrenal gland. Development 139, 4561-4570 (2012)

28. Francis, J. C., Thomsen, M. K., Taketo, M. M. \& Swain, A. beta-catenin is required for prostate development and cooperates with Pten loss to drive invasive carcinoma. PLoS Genet. 9, e1003180 (2013).

29. Ragazzon, B., Lefrancois-Martinez, A. M., Val, P., Tournaire, C., Berger, M., Gachancard-Bouya, J. L. et al. ACTH and PRL sensitivity of highly differentiated cell lines obtained by adrenocortical targeted oncogenesis. Endocr. Res. 30, 945-950 (2004).

30. Morgan, R., Pirard, P. M., Shears, L., Sohal, J., Pettengell, R. \& Pandha, H. S. Antagonism of HOX/PBX dimer formation blocks the in vivo proliferation of melanoma. Cancer Res. 67, 5806-5813 (2007).

31. Subramanian, A., Tamayo, P., Mootha, V. K., Mukherjee, S., Ebert, B. L., Gillette, M. A. et al. Gene set enrichment analysis: a knowledge-based approach for interpreting genome-wide expression profiles. Proc. Natl Acad. Sci. USA 102, 15545-15550 (2005)

32. Wassef, M., Rodilla, V., Teissandier, A., Zeitouni, B., Gruel, N., Sadacca, B. et al. Impaired PRC2 activity promotes transcriptional instability and favors breast tumorigenesis. Genes Dev. 29, 2547-2562 (2015).
33. Val, P., Martinez-Barbera, J. P. \& Swain, A. Adrenal development is initiated by Cited 2 and $\mathrm{Wt} 1$ through modulation of $\mathrm{Sf}-1$ dosage. Development 134, 2349-2358 (2007)

34. Nogueira, E. F., Vargas, C. A., Otis, M., Gallo-Payet, N., Bollag, W. B. \& Rainey, W. E. Angiotensin-ll acute regulation of rapid response genes in human, bovine, and rat adrenocortical cells. J. Mol. Endocrinol. 39, 365-374 (2007).

35. Batisse-Lignier, M., Sahut-Barnola, I., Tissier, F., Dumontet, T., Mathieu, M., Drelon, C. et al. P53/Rb inhibition induces metastatic adrenocortical carcinomas in a preclinical transgenic model. Oncogene 36, 4445-4456 (2017).

36. Alharbi, R. A., Pettengell, R., Pandha, H. S. \& Morgan, R. The role of HOX genes in normal hematopoiesis and acute leukemia. Leukemia 27, 1000-1008 (2013).

37. Audenet, F., Mejean, A., Chartier-Kastler, E. \& Roupret, M. Adrenal tumours are more predominant in females regardless of their histological subtype: a review. World J. Urol. 31, 1037-1043 (2013).

38. Grabek, A., Dolfi, B., Klein, B., Jian-Motamedi, F., Chaboissier, M. C. \& Schedl, A. The adult adrenal cortex undergoes rapid tissue renewal in a sex-specific manner. Cell Stem Cell 25, 290-296 (2019).

39. Dumontet, T., Sahut-Barnola, I., Septier, A., Montanier, N., Plotton, I., RoucherBoulez, F. et al. PKA signaling drives reticularis differentiation and sexually dimorphic adrenal cortex renewal. JCl Insight 3, e98394 (2018).

40. Lee, F. Y., Faivre, E. J., Suzawa, M., Lontok, E., Ebert, D., Cai, F. et al. Eliminating SF-1 (NR5A1) sumoylation in vivo results in ectopic hedgehog signaling and disruption of endocrine development. Dev. Cell 21, 315-327 (2011).

41. Zubair, M., Oka, S., Parker, K. L. \& Morohashi, K. Transgenic expression of Ad4BP/ SF-1 in fetal adrenal progenitor cells leads to ectopic adrenal formation. Mol. Endocrinol. 23, 1657-1667 (2009).

42. Syu, J. S., Baba, T., Huang, J. Y., Ogawa, H., Hsieh, C. H., Hu, J. X. et al. Lysosomal activity maintains glycolysis and cyclin E1 expression by mediating Ad4BP/SF1 stability for proper steroidogenic cell growth. Sci. Rep. 7, 240 (2017).

43. Li, Y., Yang, X. H., Fang, S. J., Qin, C. F., Sun, R. L., Liu, Z. Y. et al. HOXA7 stimulates human hepatocellular carcinoma proliferation through cyclin E1/CDK2. Oncol. Rep. 33, 990-996 (2015).

44. Basham, K. J., Rodriguez, S., Turcu, A. F., Lerario, A. M., Logan, C. Y., Rysztak, M. R. et al. A ZNRF3-dependent Wnt/beta-catenin signaling gradient is required for adrenal homeostasis. Genes Dev. 33, 209-220 (2019).

45. Wellik, D. M., Hawkes, P. J. \& Capecchi, M. R. Hox11 paralogous genes are essential for metanephric kidney induction. Genes Dev. 16, 1423-1432 (2002).

46. Yan, Z. C., He, L., Qiu, J. H., Deng, W. T., Lu, J. R., Yuan, Z. et al. LncRNA HOTAIR participates in the development and progression of adrenocortical carcinoma via regulating cell cycle. Eur. Rev. Med Pharm. Sci. 22, 6640-6649 (2018).

47. Morgan, R., El-Tanani, M., Hunter, K. D., Harrington, K. J. \& Pandha, H. S. Targeting HOX/PBX dimers in cancer. Oncotarget 8, 32322-32331 (2017).

(i) Open Access This article is licensed under a Creative Commons Attribution 4.0 International License, which permits use, sharing, adaptation, distribution and reproduction in any medium or format, as long as you give appropriate credit to the original author(s) and the source, provide a link to the Creative Commons license, and indicate if changes were made. The images or other third party material in this article are included in the article's Creative Commons license, unless indicated otherwise in a credit line to the material. If material is not included in the article's Creative Commons license and your intended use is not permitted by statutory regulation or exceeds the permitted use, you will need to obtain permission directly from the copyright holder. To view a copy of this license, visit http://creativecommons. org/licenses/by/4.0/.

(c) The Author(s) 2020 